\title{
Bullous pyoderma gangrenosum as a predictor of hematological malignancies $^{*}$
}

\author{
Aldana Soledad Vacas ${ }^{1}$ \\ Ana Clara Torre ${ }^{1}$
}

\author{
María Luz Bollea-Garlatti ${ }^{1}$ \\ Ricardo Luis Galimberti
}

DOI: http:/ /dx.doi.org/10.1590/abd1806-4841.20187031

\begin{abstract}
Pyoderma gangrenosum may precede, coexist or follow diverse systemic diseases. The bullous variety is usually associated with hematologic disorders. From 31 patients with pyoderma gangrenosum diagnosed in our hospital during 10 years, only 2 presented with the bullous type. One patient had previous diagnosis of chronic myeloid leukemia. Both had fast-progressive, painful lesions, and both showed an excellent response to systemic corticosteroids. In less than 3 months the lesions recurred simultaneously with the progression of chronic myeloid leukemia in one patient and with the initial diagnosis of acute myeloid leukemia in the other one. They died in less than four weeks. These findings emphasize the importance of its timely diagnosis. Cutaneous lesions could be the first predictor of an underlying disease.
\end{abstract}

Keywords: Leukemia; Leukemia, myeloid; Pyoderma gangrenosum

\section{INTRODUCTION}

Pyoderma gangrenosum (PG) is an inflammatory, necrotizing disease of rapid progression. It is a painful and invalidating condition that belongs to the group of the neutrophilic dermatosis. ${ }^{1}$

PG is usually associated with systemic diseases such as inflammatory bowel disease (IBD), arthritides, hematological disorders, and less frequently with HIV, hepatitis C virus infection, systemic lupus erythematosus, diabetes mellitus and psoriasis. ${ }^{1-5}$

There are different clinical variants of PG: ulcerous, bullous, pustular and vegetative. Bullous PG is characterized by the presence of round, very painful blisters with serous or hemorrhagic content that have rapid progression and tend to evolve into necrosis. This atypical type is the clinical manifestation that most frequently develops along with hematological diseases, such as myelogenous leukemia, lymphoma, monoclonal gammopathy, and myelodysplastic syndrome. ${ }^{2-4}$ It is estimated that $70 \%$ of the cases precede, accompany or follow hematologic malignancies. ${ }^{2,6-8}$

In order to evaluate the characteristics of bullous PG in a time frame of ten years, we reviewed the clinical records of patients diagnosed with PG between September 1st, 2004 and August 31st, 2014 at our hospital. From 31 patients with PG, only 2 presented with the bullous type.

\section{CASE REPORTS}

Case 1. A 42-year-old female patient had a 24-hour fever and multiple tense, fluid-filled blisters, with surrounding erythema, located on the lower lip and on the lower limbs (Figure 1). She also had erosions covered by necrotic crusts on the face and on the upper limbs. In addition, she had painful pustules on the face.

The blood and skin cultures were negative. The histopathology showed the presence of dense neutrophilic infiltration in the dermis, which supported the clinical diagnosis of bullous PG.

The patient received methylprednisolone $500 \mathrm{mg} /$ day intravenously for 3 days and then continued with oral prednisone 40 $\mathrm{mg} /$ day. The lesions dried and re-epithelialization was complete in three weeks.

After 3 months, the patient developed new skin lesions. On that occasion, laboratory test showed anemia, thrombocytopenia and leukocytosis $\left(114,500\right.$ leukocyte $\left./ \mathrm{mm}^{3}\right)$ with blasts cells $(65 \%)$ in peripheral blood. The bone marrow biopsy diagnosed acute myeloid leukemia.

The patient died of sepsis 4 weeks after diagnosis.

Case 2. A 66-year-old male patient with chronic myeloid leukemia, was hospitalized with fever, hypotension and a $15 \times 12$ $\mathrm{cm}$ oval erythematous plaque, above which there was a large hem-

Received on 16.02.2017.

Approved by the Advisory Board and accepted for publication on 30.03.2017.

Work performed at the Hospital Italiano de Buenos Aires - Cdad. Autónoma de Buenos Aires, Argentina.

Financial support: None.

Conflict of interest: Prof. Galimberti is a senior researcher at Novartis, Eli Lilly and AbbVie.

Department of Dermatology at Hospital Italiano de Buenos Aires - Buenos Aires, Argentina.

Department of Dermatology at Universidad de Buenos Aires - Buenos Aires, Argentina. 


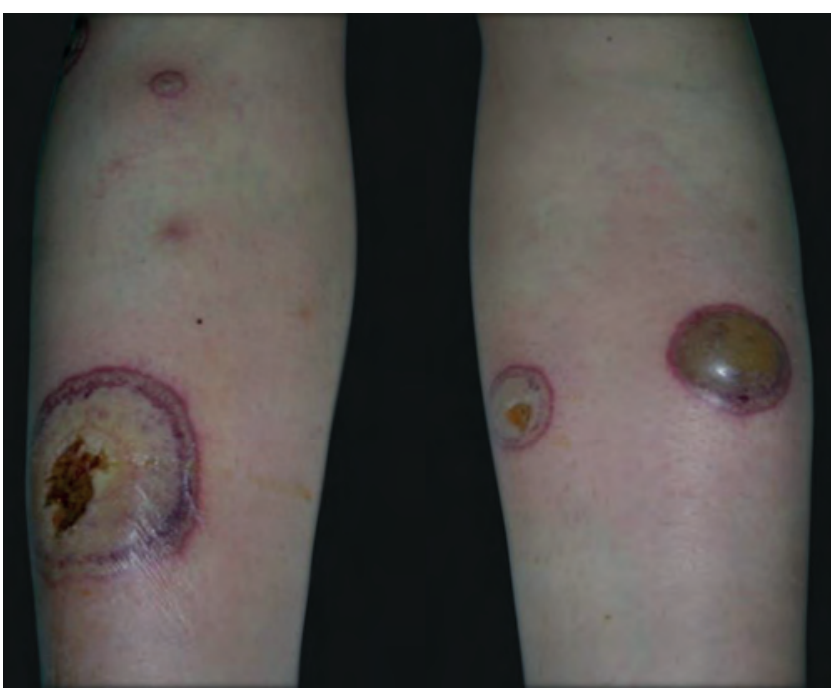

FIGURE 1: Multiple tense, fluid-filled blisters of variably sized, with surrounding erythema, located on the lower limbs

orrhagic bulla. The lesion was very painful and it was located in the lower third of the right leg (Figure 2).

Laboratory tests showed anemia, thrombocytopenia and leukocytosis $\left(20,210\right.$ leukocytes $\left./ \mathrm{mm}^{3}\right)$, which were expected due to the underlying disease, and neutrophilia (87.5\%). We performed blood and skin cultures, which turned out negative. The histological study of the skin lesion showed a diffuse neutrophilic infiltrate in the dermis.

We initiated treatment with oral prednisone $100 \mathrm{mg} /$ day, reducing the fever and the pain in 24 hours. We observed a complete re-epithelialization of the lesion in 9 weeks.

One month later, the patient developed fever and a recurrence of pyoderma gangrenosum above the residual scarring. The laboratory tests showed progression of the underlying disease (58,530 leukocytes $/ \mathrm{mm}^{3}, 82 \%$ neutrophils). The patient evolved to severe sepsis and died ten days later.

\section{DISCUSSION}

PG is a necrotizing neutrophilic dermatosis which belongs

\section{REFERENCES}

1. Marzano AV, Fanoni D, Antiga E, Quaglino P, Caproni M, Crosti C, et al. Expression of cytokines, chemokines and other effector molecules in two prototypic autoinflammatory skin diseases, pyoderma gangrenosum and Sweet's syndrome. Clin Exp Immunol. 2014;178:48-56.

2. Su WP, Davis MD, Weenig RH, Powell FC, Perry HO. Pyoderma gangrenosum: clinicopathologic correlation and proposed diagnostic criteria. Int J Dermatol. 2004:43:790-800.

3. Lois M, Pizzariello G, Olivares L, Maronna E. Estudio retrospectivo de pacientes con pioderma gangrenoso en un período de 20 años y revisión de la literatura. Dermatol. Argent. 2012;18:24-9.

4. Sakiyama M, Kobayashi T, Nagata Y, Fujimoto N, Satoh T, Tajima S. Bullous pyoderma gangrenosum: a case report and review of the published work. $J$ Dermatol. 2012;39:1010-5.

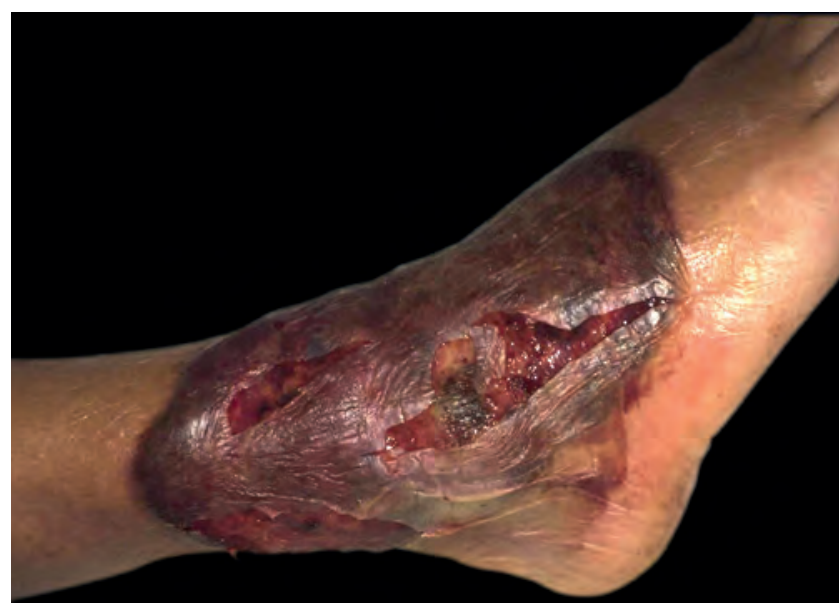

FIGURE 2: 15 x 12cm, oval erythematous plaque, above which there was a large hemorrhagic bulla. The lesion was very painful and it was located on the lower third of the right leg

to the group of autoinflammatory diseases. ${ }^{1}$ It may precede, coexist or follow diverse systemic diseases. The bullous variety is not frequent and it is usually associated with hematologic disorders ${ }^{4-8}$

Two of the 31 patients diagnosed with PG in our department during 10 years, had the bullous type. Both of them were associated with myeloid leukemia. In case number 1, the PG preceded leukemia for four months and relapsed simultaneously. In case number 2, the patient had been diagnosed with leukemia and developed PG with the progression of the underlying disease.

We want to highlight the excellent response of the patients to corticosteroid therapy. In less than 24 hours, the pain and the progression of the lesions stopped. However, in less than three months, they presented PG recurrence, simultaneous to the emergence of leukemia in our first patient and to the progression of the underlying disease in the second patient.

Bullous PG associated with hematologic malignancies often responds positively to chemotherapy. ${ }^{6,8}$ However, in these cases, we could not find the specific treatment for their hematologic diseases because both patients had multiple complications during hospitalization, and died in less than 4 weeks from the diagnosis of leukemia. $\square$

How to cite this article: Vacas AS, Bollea-Garlatti ML, Torre AC, Galimberti RL. Bullous pyoderma gangrenosum as a predictor of hematological malignancies. An Bras Dermatol. 2018;93(1):133-4. 